\title{
GROWTH INHIBITION IN THE SKIN INDUCED BY PARENTERAL ADMINISTRATION OF ADRENOCORTICOTROPIN
}

\author{
BURTON L. BAKER, DWIGHT J. INGLE, CHOH H. LI AND \\ HERBERT M. EVANS \\ Department of Anatomy, University of Michigan Medical School, Ann Arbor; \\ Research Laboratories, The Upjohn Company, Kalamazoo, Michigan \\ and Institute of Experimental Biology, University of \\ California, Berkeley \\ SEVENTEEN FIGURES
}

For a number of years the anterior hypophysis has been known to play an important rôle in the regulation of body growth. In general, the effects of hypophyseal secretions on growth have been found to be of a stimulative character. The growth stimulating or inhibiting action of such hormones is demonstrated most precisely by the changes they elicit in those tissues which normally undergo the most rapid cell division. Hair is such a rapidly growing structure and its growth has been shown to be affected by the anterior hypophysis. For example, Smith ('30) demonstrated in the rat that pituitary activity is essential to the normal maturation of hair from the infantile to the adult type. Apparently the growth hormone is primarily responsible for this effect (Thompson and Gaiser, '32). Microscopically, hypophysectomy of young rats has been found to cause a general atrophy of the skin with reduction in size of connective tissue fibers, hair follicles and their papillae (Snow and Whitehead, '35). These changes were reversed by treatment of hypophysectomized rats with extracts containing the growth hormone. The general conclusion to be drawn from these early experiments 
is that the growth hormone stimulates the hair to grow and mature.

The discovery that growth of hair is accelerated by adrenalectomy (Butcher and Richards, '39; Butcher, '41; Ralli and Graef, '43) and that this rapid growth after adrenalectomy may be inhibited by the administration of cortical preparations indicates that the adrenal cortex normally acts either directly or indirectly as an inhibitor of hair growth. Since the secretory activity of at least part of the adrenal cortex is controlled by the anterior hypophysis, it is pertinent to investigate the manner in which the anterior hypophysis affects the growth of hair when it acts through the adrenal cortex. This problem is unusually challenging in view of the evidence just cited which indicates that the adrenal cortex inhibits hair growth. Is it possible that the anterior hypophysis possesses the capacity for both stimulating and inhibiting hair growth - stimulating it by direct action of the growth hormone and inhibiting it by accelerating the release of cortical steroids? Might it have some influence also on other cutaneous structures which exhibit continuous or intermittent cell division such as the epidermis and sebaceous glands? The development of a method for preparation of hypophyseal adrenocorticotropic hormone in a comparatively pure form by Li, Evans and Simpson ('43) makes possible an investigation of the action of the anterior hypophysis on the skin when it acts by way of first stimulating the adrenal cortex. This study, in which Doctor Li's preparation has been used attempts to answer the questions which have been raised.

\section{MATERIALS AND METHODS}

The experimental procedures employed in this study have been described in detail elsewhere (Baker, Ingle, Li and Evans, '48). In summary, adult male rats of the Sprague-Dawley strain were force-fed diets high in carbohydrate, fat or protein. Following a period of adaptation to this type of feeding, they were injected with adrenocorticotropin. In experiment 1 , one rat on each of the three diets received $1 \mathrm{mg}$ of adrenocortico- 
tropin per day for 21 days, with the same number of animals serving as controls. Experiment 2 was similar except that the dosage of hormone was increased to $3 \mathrm{mg}$ per day. In experiment $3,8 \mathrm{mg}$ of adrenocorticotropin were injected daily for 10 days into two rats on the high fat and high protein diets with two controls on each diet; on the high carbohydrate diet, 4 animals were injected with the hormone and there were two controls. The amount of adrenocorticotropin administered daily was divided into 8 doses which were injected at intervals of two hours. The control animals were given injections of $0.9 \%$ sodium chloride at the same times.

At autopsy samples of skin measuring about $1 \times 0.5 \mathrm{~cm}$ were removed from the dorsum of the body in the thoracic region. This method of sampling did not take into account the waves and cycles of hair growth which occur in the rat (Butcher, '34). Therefore, in order to obviate the possibility that the skins of treated rats which showed no hair growth might have been taken from normally inactive arcas and those of the controls from normally active areas, experiment 4 was carried out with three rats receiving $3 \mathrm{mg}$ of adrenocorticotropin daily and three others serving as controls. This procedure duplicated the conditions of experiment 2, except that all of these rats were fed a medium carbohydrate diet (Ingle, Nezamis and Prestrud, '47). Since it has been reported by Butcher ('34) that hair in rats grows in waves which begin mid-ventrally and proceed dorsally, a strip of skin $4 \mathrm{~mm}$ wide and extending from the mid-ventral to mid-dorsal line was excised from the lumbar region of each rat at autopsy. It was sectioned in such a way that a complete microscopic picture of the skin was thus available for one-half the circumference of the body.

The skin samples were fixed in Bouin's fluid and imbedded in paraffin. In the case of the samples removed from the dorsum of the back, approximately 2.6 to $3 \mathrm{~mm}$ of skin in the paraffin blocks was sectioned serially and studied. The stains used were Harris' hematoxylin and eosin, Regaud's iron hematoxylin and Masson and the Weigert procedure for 
elastic fibers. In experiment 4, small pieces of abdominal skin were fixed in chilled $80 \%$ alcohol and prepared for the study of alkaline phosphatase by the method of Gomori ('39); other pieces were fixed in $4 \%$ basic lead acetate and stained metachromatically with toluidine blue for demonstration of mast cells.

In the earlier experiments no attempt was made to study the re-growth of hair on shaved areas during treatment. However, in experiment 4 the hair was clipped from the entire body at the beginning of the experiment. Then, at weekly intervals during treatment, records were made of the extent of hair growth during the preceding week by sketching the growth pattern and the hair was clipped again.

\section{OBSERVATIONS}

Comparison of figures 1 and 3 with figures 2 and 4 demonstrates that adrenocorticotropin caused an apparent thinning of the skin. However, closer scrutiny reveals that this appearance resulted largely from the reduction in thickness of the subcutaneous layer of loose fibro-elastic connective tissue and fat (panniculus adiposus), a change so marked as to be readily discernible upon macroseopic inspection of the stained slide. In general, the panniculus adiposus was obliterated completely in which case a dense mass of collagenous fibers approximated directly the panniculus carnosus muscle. In other rats the reduction was not as great and narrowed patches of fat and loose connective tissue remained in some localities especially around whatever hair follicles might still be present in this region (fig. 4). The effect seemed to be greatest following the $3 \mathrm{mg}$ daily dose administered for 21 days and less when $1 \mathrm{mg}$ was given daily for this period or $8 \mathrm{mg}$ for the shorter period of 10 days.

Microscopic measurement of the dermis demonstrated that the thickness of this layer of the skin proper was not altered significantly by either diet or treatment with the hormone. It should be mentioned in this connection that accurate measure- 
ment was difficult because of the irregularity of the boundary between the dermis and panniculus adiposus.

Epidermis. Dorsally in the control animals a definite layering of the epidermis was evident with stratum germinativum, corneum and, at least, a discontinuous stratum granulosum being present constantly. Occasionally a deeply eosinophilic layer could be seen external to the stratum granulosum which resembled somewhat the stratum lucidum of human skin. The stratum germinativum and, in places, the stratum granulosum were several cell layers in thickness (fig. 5). The epidermis became progressively thinner with a less prominent stratum granulosum toward the ventral side of the body.

Treatment with adrenocorticotropin caused a striking change in the surface contour of the skin. Whereas in the control rats this surface was rather even and smooth especially in the dorsal area (figs. 1 and 3 ) following treatment the epidermis was thrown up into papilla-like folds giving it a "crinkly" appearance on microscopic observation (figs. 2, 8). This alteration was accompanied by a thinning of the epidermis which was most striking dorsally because in this region the epidermis normally attains its greatest thickness. In some locations the cellular part was reduced to one or two layers in thickness with considerable flattening of the cells (figs. 5, 6). The greatest change seemed to have occurred in the stratum germinativum and stratum granulosum. The atrophy of the former layer could have been incident to an inhibition of cellular proliferation. Particularly outstanding was the great reduction in size and number of the keratohyalin granules in the stratum granulosum (fig. 6). Assuming these granules to represent one stage in the formation of keratin, this alteration might be interpreted as being indicative of a defect in the process of cornification. Indeed, in some rats which were affected by the treatment more markedly, the atrophic epidermis possessed a comparatively thin stratum corneum (fig. 6). However, the observer could not always ascertain how much the stratum corneum had been altered by the experimental conditions because of the impossibility of assessing the extent 
to which the layer had been disturbed by mechanical attrition. In such atrophic epidermis no eosinophilic layer which might be regarded as a stratum lucidum was present. All of these changes were found consistently on the $3 \mathrm{mg}$ dose except in one of the 6 treated rats in which the epidermis was not significantly thinner than that of its control. These effects occurred variably on the 1 and $3 \mathrm{mg}$ dosage.

Hair. The full magnitude of the extent to which adrenocorticotropin inhibited growth of hair could not be appreciated from the small pieces of skin which were removed from the back in the first experiments. The reliability of comparisons made by this procedure between the rate and extent of hair growth in a control and an experimental rat is dependent upon the expectation that the pattern of hair growth, except for the hormone treatment, would have been identical in both animals. One could not validly make such an assumption, especially since the control and experimental animals in our studies were not litter mates. Nevertheless, in these specimens suggestive evidence of retarded development of hair follicles and bulbs due to treatment with adrenocorticotropin was obtained.

This repression seemed to be related directly to the dosage and duration of treatment. On the $1 \mathrm{mg}$ dose for 21 days, there was evidence of inhibition following treatment with the hormone in only the animal on the carbohydrate diet in which case no follicles were found to extend deeper than the middle of the dermis in the serial sections which were studied. With increase of the dosage to $8 \mathrm{mg}$ for the shorter period of 10 days, inhibition of hair growth became more definite. In one adrenocorticotropin-protein treated rat there was no effect. However, in all of the other rats, there were fewer follicles which reached to the panniculus adiposus and, in general, the greater proportion of those which did were atrophic (figs. 3 and 4). This stood in contrast to the controls in which more of the hairs possessed large growing bulbs.

Increasing the daily dose to $3 \mathrm{mg}$ for the longer period of 21 days resulted in still more impressive evidence of inhibition 
of hair growth. In the serial sections of the skin of the three animals in experiment 2 treated at this dosage, in only one rat (carbohydrate diet) did a rare follicle reach the panniculus adiposus and the few which did, possessed bulbs far smaller than those in the controls. In the other two treated rats, no follicles were found to extend beyond the middle of the corium (fig. 2). In contrast, in all of the controls numerous follicles extended to the panniculus adiposus and many of these possessed large bulbs (fig. 1).

In experiment 4 on the $3-\mathrm{mg}$ daily dosage any doubt as to the marked inhibitory effect of adrenocorticotropin on hair growth was removed. Re-growth of hair was observed grossly on these animals. Treatment with adrenocorticotropin had no apparent effect during the first two weeks of the experiment. However, at the end of three weeks there was intense inhibition of hair growth in the treated rats as compared with the controls (figs. 13 and 14). During the third week, hair growth had occurred in the latter animals over almost the entire thoracico-lumbar region. In contrast, only thin, sparse hair had grown in small areas of the skin of each of the three adrenocorticotropin-treated rats. Macroscopic observation indicated that variation of the diet did not modify the inhibitory action of adrenocorticotropin on the growth of hair.

Microscopic study of a strip of skin from one-half the circumference of the body in these rats offered confirmatory evidence of the markedly reduced rate of hair growth. In the three controls, hair was growing throughout most of the strip as shown by the presence of many large active bulbs in the panniculus adiposus. This microscopic picture confirmed the previously described gross observations which were made on these animals. In contrast, two of the three treated rats were almost completely inactive with only an occasional hair bulb being found and most of these were small and not surrounded by fat. In the third treated rat, the amount of inhibition was still very great but in this animal, hair was growing through about one-twentieth or less of the strip of skin. In its control, growing bulbs were found almost throughout the entire strip. 
In samples of skin taken from rats treated with adrenocorticotropin, epithelial buds presaging a period of active hair growth could be found at the end of some of the dormant hair follicles. These clusters of cells, however, appeared to be in an atrophic condition since the cells were small and nuclei densely packed (fig. 15). Additional evidence of inactivity is found in the absence of alkaline phosphatase in or around such cells (fig. 17). Johnson, Butcher and Bevelander ('45) demonstrated small amounts of this enzyme in the epithelial hair buds and large amounts in the surrounding connective tissue of growing hair in newborn rats. In our control specimens, growing epithelial buds showed intense phosphatase activity as did the surrounding connective tissue. As the epithelial buds expanded into large hair-forming bulbs the enzyme was most concentrated in the connective tissue papilla and sheath of the follicle and, frequently, in the periphery of the epithelial part of the bulb (fig. 16).

Sebaceous glands. Since sebaceous glands are of a holocrine type, their epithelial cells proliferate continuously in order to replace those which are converted into and secreted as droplets of oil. It might be expected that a growth-inhibiting substance such as adrenocorticotropin would inhibit this proliferation and, thus, cause an atrophy of the gland. Some difficulty is attendant upon the determination of such effects since in any histological section of skin, the sebaceous glands normally vary in size and distribution. Therefore, only extreme changes are detected easily. It did seem clear that on the $1 \mathrm{mg}$ dosage no effect could be discerned in the size or number of these structures. However, in the small dorsal skin samples from rats on the 3-and 8-mg dosages, there was in all cases a variable reduction in size of the glands but it was not possible to be certain of a siginficant reduction in number although this was indicated in a few cases. Comparison of figures 7 and 8 illustrates the maximum evidence of sebaceous gland atrophy which was obtained by such a sampling method. Study of the long strips of skin from the rats in experiment 4 indicated that the effect of adrenocortico- 
tropin on the sebaceous glands was not as great as was deduced from the earlier studies. In the treated animals one could find some glands of an approximately normal size even though, in general, they were probably somewhat smaller. Therefore, it is to be concluded that under the conditions of these experiments, the atrophy of the sebaceous glands induced by adrenocorticotropin did not compare with the changes induced in the epidermis or hair. The observation that considerable alkaline phosphatase activity was still evident in these glands after treatment with the hormone (fig. 17) further suggests that their function had not been suppressed greatly.

Dermis. Adrenocorticotropin caused a variable increase in compactness of arrangement of the constituent collagenous fibers of the dermis which in most animals was very striking. This change seemed to be accompanied by a swelling of the collagenous fibers with a parallel reduction in the intervening tissue spaces (figs. 11 and 12). Mast cells as revealed by toluidine blue, were still present after treatment with adrenocorticotropin but were probably reduced somewhat in number.

Elastic fibers in the control animals were most numerous in the upper one-third to one-half of the corium, around hair follicles and sebaceous glands and beneath the panniculus carnosus muscle. There was no significant change induced in them by adrenocorticotropin (figs. 9 and 10). However, in some treated rats the fibers may have been slightly more coarse as well as more concentrated around the hair follicles. The latter effect probably was incident to the atrophy of the hair follicles.

\section{DISCUSSION}

In an investigation of the relationship of the hypophysis and adrenal cortex to the regulation of growth, it is significant that in these experiments hypophyseal adrenocorticotropin caused a reduction in size of the epithelial integumentary structures which exhibit more or less continuous cellular division, this atrophy being demonstrated by the epidermis, the 
growing parts of hair and less significantly by the sebaceous glands. These observations extend the earlier ones of Moon ('37) who reported a greatly retarded growth of hair over the shaved areas of immature gonadectomized rats injected with large doses of crude adrenocorticotropic extract. It would appear that these findings fit into the general pattern of growth inhibition which apparently follows treatment with adrenocorticotropin. All of the animals in this experiment which received injections of the hormone lost weight except those on the protein diet. Nevertheless, these latter rats gained less weight than their controls (Ingle, Prestrud, Li and Evans, '47). Adrenocorticotropin has been shown to inhibit body growth (Evans, Simpson and Li, '43), proliferation of the epiphyseal cartilage (Becks, Simpson, Li and Evans, '44) and to antagonize the action of the growth hormone (Marx, Simpson, Li and Evans, '43; Becks, Simpson, Marx, Li and Evans, '44). Marx et al. ('43) showed that diminished food consumption did not explain the growth inhibition caused by adrenocorticotropin. This factor was eliminated in our experiment by force-feeding the animals. It is also possible that suppressed cellular proliferation may be a factor in the profound atrophy of the thymus which we found to follow treatment with adrenocorticotropin.

In the studies of Evans and his collaborators just cited, inhibition of growth did not ensue following treatment of adrenalectomized animals with adrenocorticotropin which demonstrates that this effect is mediated by the adrenal glands. It may be presumed that in our experiments the atrophy of the epidermis and its derivatives was brought about in a similar manner. Furthermore, urinalyses in these animals (Ingle, Prestrud, Li and Evans, '47) gives suggestive evidence as to the specific steroids whose release by the adrenal cortex may be stimulated by adrenocorticotropin. All of the rats treated with adrenocorticotropin showed an increased non-protein nitrogen excretion and some of those concurrently fed high and medium carbohydrate diets exhibited glycosuria. Such effects are characteristic actions of the 11- 
oxysteroids. Thus, it appears possible that adrenocorticotropin caused the secretion by the adrenal cortex of C-11 oxygenated steroids which in turn inhibited growth by promoting the catabolism of protein. Evidence for the inhibition of growth by these steroids will be summarized subsequently (Baker and Whitaker, this journal).

The changes brought about in the connective tissue of the dermis by adrenocorticotropin raise an important question as to how this effect may be involved in the inhibition of growth of the epithelial structures in the skin. Is it possible that the primary effect of adrenocorticotropin is on the connective tissue and that the epithelial atrophy is secondary? One might expect that structural modification of the dermis could have impaired the nutrition of the epidermis, hair and sebaceous glands either by compression of the small blood vessels or by interfering with the functioning of the tissue fluid. A satisfactory evaluation of the state of the vascular bed by microscopic study of our preparations proved to be impossible. Apparent swelling of collagenous fibers is suggestive of a disturbance in the electrolytes of the tissue fluids. Ingle, Prestrud, Li and Evans ('47) observed an increased excretion of potassium in these rats to be induced by adrenocorticotropin. Some loss of sodium and chloride occurred during the first two days of treatment but this effect was not regarded as significant. At the present time one cannot evaluate the proper place of connective tissue in the chain of events leading to suppression of growth by adrenocorticotropin.

The atrophy of the panniculus adiposus is significant in view of the fatty infiltration of the liver which occurred in those rats which were treated with adrenocorticotropin and fed a high carbohydrate diet (Baker et al., '48). In this report two possible explanations were offered for the accumulation of fat in the liver: (1) interference with carbohydrate metabolism in the liver with conversion of the carbohydrate to fat and deposition of it in the liver and (2) interference with the transport of fat between the body depots and the liver. If the latter explanation is a factor in the accumulation 
of fat in the liver after treatment with adrenocorticotropin, then the marked atrophy of this extensive depot (panniculus adiposus) becomes of utmost importance.

The presence or absence of a well-developed panniculus adiposus must bear some relation to the growth status of hair. However, the exact nature of this relationship has not been clarified. Butcher ('34) concluded that the presence of a well-developed fatty layer is not prerequisite to the growth of hair. Nevertheless, he observed the resting condition of the hair to be followed by a reduction in fatty content of the subcutaneous layer. It was his opinion that the presence of fat facilitated more rapid growth of hair.

Finally, it seems that some of the characteristics of skin after treatment with adrenocorticotropin are similar to those of normal skin which is in a state of inactive hair growth. The reduction in the panniculus adiposus and the undeveloped state of the hair follicles fits into this category. However, the increased compactness of the dermis and atrophy of the epidermis appeared to be more extreme modifications than occur in the skin concurrently with the cessation of hair growth. Our procedures did not permit an analysis of the true nature of the connective tissue changes.

\section{SUMMARY}

Adult male rats were force-fed high carbohydrate, high fat, high protein or medium carbohydrate diets and treated with adrenocorticotropin at daily dose levels of 1 or $3 \mathrm{mg}$ for 21 days and $8 \mathrm{mg}$ for 10 days. As a result of treatment with this hormone, there occurred thinning of the epidermis, reduced growth of hair, an inconsistent reduction in size of the sebaceous glands, increased compactness of the dermal connective tissue and reduction of the panniculus adiposus. Dosage and duration of treatment seemed to be important factors in eliciting these responses, the most marked effect being obtained with the 3-mg daily dose administered for 21 days. Variation of the diet did not modify the response to the hormone. 


\section{LITERATURE CITED}

Baker, B. L., D. J. Ingle, C. H. Li ANd H. M. Evans 1948 The effect on liver structure of treatment with adrenocorticotropin under varied dietary conditions. Am. J. Anat., 8Q: 75-103.

Becks, H., M. F. Simpson, C. H. Li AND H. M. Evans 1944 Effects of adrenocorticotropic hormone (ACTH) on the osseous system in normal rats. Endocrin., 34: 305-310.

Broks, II., M. E. Simpson, W. Marx, C. H. Li and II. M. Evans 1944 Antagonisin of pituitary adrenocorticotropic hormone (ACTH) to the action of growth hormone on the osseous system of hypophysectomized rats. Endoerin., 34: 311-316.

Butrerr, F. O. 1934 The hair eycles in the albino rat. Anat. Ree., 61: 5-20. 1.94. Accelerated hair growth in the rat after adrenalectomy cannot he attributed to the thyroid. Proc. Soc. Exp. Biol, and Med., 48: 120122.

Butcher, F. O., AND R. A. Richards 1939 The relation of the adrenals to the retarded hair growth in underfed albino rats. Endoerin., $25: 787-792$.

Evans, H. M., M. E. Simpson ANd C. H. Lr 1943 Inhibiting effect of adrenocorticotropic hormone on the growth of male rats. Endocrin., 33: 237238.

Gomori, G. 1939 Mierotechnical demonstration of phosphatase in tissue sections. Proc. Soc. Exp. Biol. and Med., 42: 23-26.

InGLe, D. J., J. E. Nezamis And M. C. Prestrud 1947 The effect of diethylstilbestrol upon alloxan diabetes in the male rat. Endocrin., 41: 207-212.

INGLE, D. J., M. C. PREstrud, O. H. LI AND II. M. Evans 1947 The relationship of diet to the effect of adrenocorticotrophic homone upon urinary nitrogen, glueose and electrolytes. Endocrin., 41: 170-176.

Johnson, P. L., E. O. Butcher AND G. Bevelander 1945 The distribution of alkaline phosphatase in the cyelie growth of the rat hair follicle. Anat. Rec., $93: 355-361$.

LI, C. H., H. M. Evans AND M. E. Simpson 1943 Adrenocorticotropic hormone. J. Biol. Chem., 149: 413-424.

Marx, W., M. E. Simpson, C. H. Li and H. M. Evans 1943 Antagonism of pituitary adrenocorticotropic hormone to growth hormone in hypophysectomizod rats. Endocrin., 33: 102-105.

MooN, H. D. 1937 Inhibition of somatic growth in eastrate rats with pituitary extracts. Proc. Soc. Exp. Biol. and Med., 37 : 34-36.

RALII, E. P., AND I. GraeF 1943 Stimulating effect of adrenalectomy on hair growth and melanin deposition in black rats fed diets adequate and defieient in the filtrate factors of vitamin B. Endocrin., 82 : 1-12.

SMITH, P. E. 1930 Hypophysectomy and a replacement therapy in the rat. Am. J. Anat., 45: 205-274.

SNow, J. S., AND R. W. WHITEHEAD 1935 Relationship of the hypophysis to hair growth in the albino rat. Endoerin., 19: 88-96.

Thompson, K. W., and D. W. Gaiser 1932 The effect of diet and pituitary growth-hormone on hypophysectomized rats. Yale J. Biol. and Med., 4: $677-690$. 
PLATF 1

\section{EXPLANATION OF FIGURES}

The specimens illustrated in figures 1-4 were fixed in Bouin's fluid, sectioned at $10 \mu$, and stained with Harris' hematoxylin and eosin. Pannieulus carnosus muscle is at the left. Figures $1-12$ are of skin samples removed from the back. $\times 28$.

1 Rat 1401, fat control. The thiek epidermis possesses a rather regular surface. Many large, growing hair bulbs reach the thick panniculus adiposus. $\times 28$.

2 Rat 1301, fat, $3 \mathrm{mg}$ adrenocorticotropin per day for 21 days. The surface is "erinkly" and the epidermis is thinner and of more variable thickness. No hair follicles reach below the upper one-third of the dermis. The dermis is more dense and the panniculus adiposus is greatly reduced. $\times 28$.

3 Rat 1201, carbohydrate control. The characteristics are similar to those illustrated in figure $1 . \times 28$.

4 Rat 1404 , carbohydrate, $8 \mathrm{mg}$ adrenocorticotropin per day for 10 days. The effects are similar to those shown in figure 2 except that they are less marked. $\times 28$. 
BAKER, INGLF, LI AND EVANS
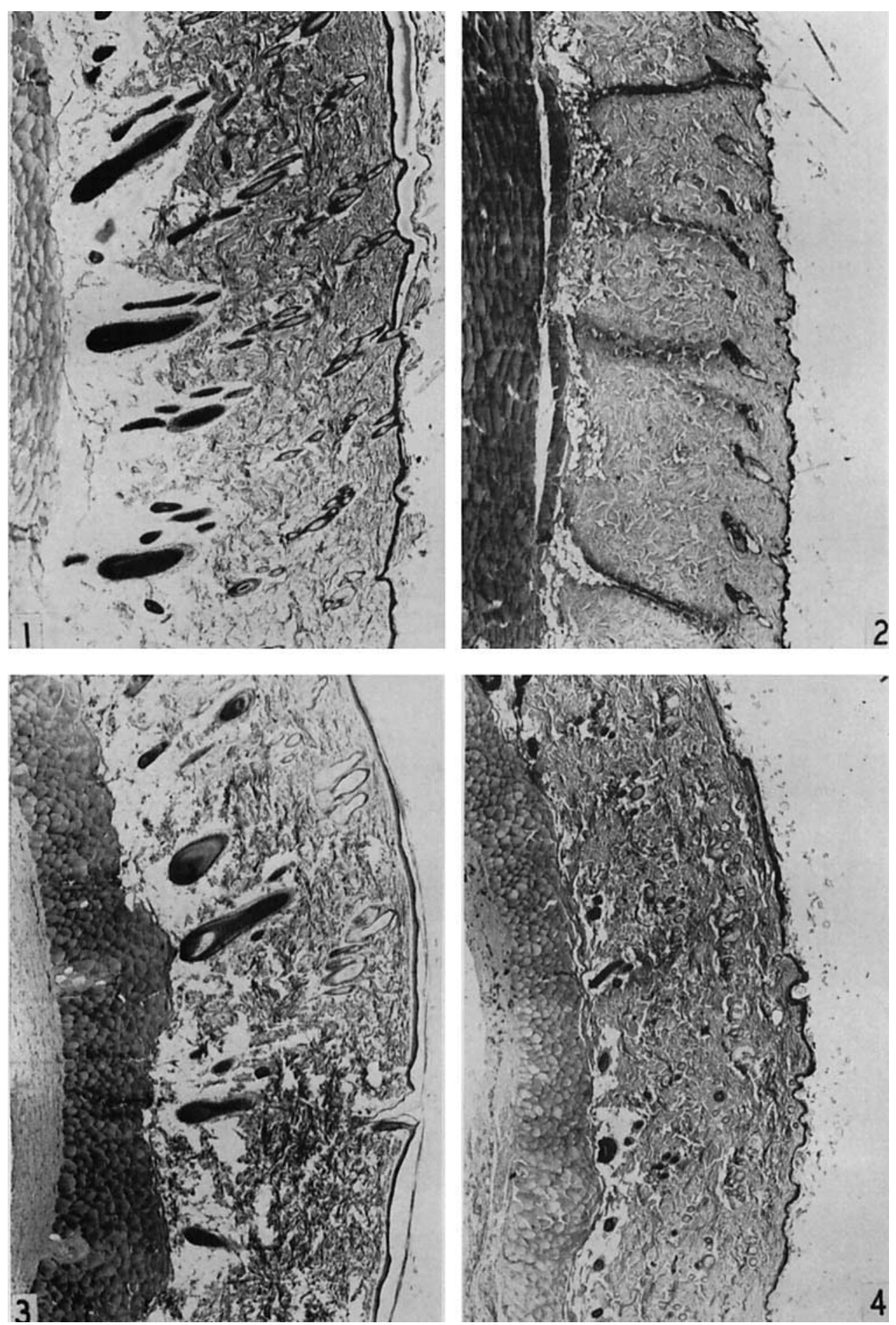
PIATE 2

EXPLANATION OF FIGURES

5 Rat 1204, carbohydrate control. The epidermis is thick with stratum germinativum, granulosum and comeum slowing distinetly. An indistinct stratum lucidum is visible above the stratum gramulosum. The keratohyalin granules (black) of the latter layer are quite prominent. $6 \mu$. Hematoxylin and eosin. $\times 464$.

6 Rat 1304, carbohydrate, $8 \mathrm{mg}$ adrenocorticotropin per day for 10 days. All strata of the epidermis are atrophic. Only a few small keratohyalin granules are present in the stratum granulosum. Technique as in figure 5.

7 Rat 1401, fat control. The epidermis is thick and smooth. Sebaceous glands are large. $10 \mu$. Hematoxylin and eosin. $\times 52$.

8 Rat 1301, fat, $3 \mathrm{mg}$ adrenocorticotropin per day for 21 days. Surface is "crinkly" and the epidermis is generally thinner and of variable thickness. Sebaceous glands are atrophic. Collagenous fibers of the dermis are swollen. Technique as in figure 7 .

9 Rat 701, protein control. The black lines in the dermis aro elastie fibers. Weigert's resorcin fuchsin with no counterstain. $\times 112$.

10 Rat 801 , protein, $1 \mathrm{mg}$ adrenocorticotropin per day for 21 days. There is no significant change in the elastic fibers. Technique as in figure 9.

11 Rat 1401, fat control. Sharply defined collagenous fibers are illustrated in the dermis with definite tissue spaces between them. A few of these spaces are widened further by technical distortion. $10 \mu$. Hematoxylin and eosin. $\times 140$.

12 Rat 1301, fat, $3 \mathrm{mg}$ adrenocorticotropin per day for 21 days. This area was selected from the same depth in the dermis as that shown in figure 11 and shows the increased compactness of the dermis with apparent swelling of collagenous fibers. Some of these spaces are also artefacts. Technique as in figure 11. 


PLAT'E 3

EXPIANATION OF HIGURES

All illustrations on this plate are of rats fed a medium earbohydrate diet.

13 Rat 18003, control; photograph taken at the termination of the experiment. General growth of hair had occurred during the third week of the experiment.

14 Rat $17003,3 \mathrm{mg}$ of adrenocorticotropin daily for 21 days. Growth of hair had been suppressed almost completely during the third week of treatment.

15 Rat $15005,3 \mathrm{mg}$ of adrenocorticotropin daily for 21 days. The epithelial bud (arrow) is composed of small densely packed nuclei. This illustrates the stage in which growth of hair was arrested by the hormone. $10 \mu$. Hematoxylin and eosin. $\times 480$.

16 Rat 16004 , control. The upper arrow points to a growing hair bulb with a central connective tissue papilla, both the papilla and epithelium giving an intensely positive reaction for alkaline phosphatase. The lower arrow points to two smaller hair buds which are likewise positive. $10 \mu$. Gomori alkaline phosphatase procedure, 24-hour incubation, $\mathrm{pH} 9.4$, sodium barbital buffer. $\times 136$.

17 Rat $15005,3 \mathrm{mg}$ adrenocorticotropin daily for 21 days. Fpithelial buds (arrows) are practically negative for alkaline phosphatase. The periphery of the sebaceous giand in the center is positive. Technique as for figure 16. 
BAKER, INGLE, LIT AND EVANS
